DE93 004851

\title{
SIMPLE OPTICS DESCRIPTION OF THE PLASMA SHEATH AND PLASMA ELECTRODE REGION \\ SHEATH AND
}

\section{L. Fink, and B. P. Curry \\ Argonne National Laboratory Argonne IL 60439}

ABSTRACT

.

A simple model of the optics of an ion source has been developed and compared with the exact Poisson solution calculated by computer code SNOW. The model replaces the Poisson potential by a Laplacian potential that is calculated by replacing the plasma and plasma sheath by a planar plate. This Laplacian potential is then used with and without a linear space-charge correction to calculate particle trajectories. In this model the only free parameter is the distance between the planar plate and the plasma electrode. In general, there is good agreement between emittance curves calculated by the model and the SNOW results even when no space-charge correction is used. This implies for the geometry we studied, that the effects of the plasma sheath and non-linear space-charge forces are small compared to aberrations introduced by the plasma aperture.

\section{INTRODUCTION}

There are a number of computer codes ${ }^{1}$ that solve (with varying levels of approximation) the poisson and Boltzman-Vlasov equations ${ }^{2}$ used to model ion sources. These codes are relatively easy to use and give a good representation of ion source performance - especially for positive ions. The major problem with these codes revolves around the difficulty in separating the significance and magnitude of the different physics effects involved in source behavior. This in turn makes the optimization of source performance more of an art than a science.

Three areas of concern in optimizing the performance of a source in terms of emittance or brightness are the shape of the plasma sheath, the aberrations introduced in the beam from the plasma aperture and extractor electrode, and non-linear space

arge effects in the region between the plasma sheath and the plasma electrode. 
Over the years there have been a number of simple physical models of the ion source. Early efforts used the concept of ion transport between two spherical segments to predict the emergence angle of the ion beam as it left the plasma electrode ${ }^{3}$. Recent efforts have modeled the growth of emittance due to space charge effects 4 , and plasma boundary curvature ${ }^{5}$.

In this paper we will examine the possibility of replacing the Poisson potential distribution with a Laplacian potential distribution rather than develop an analytical model. Since space-charge effects can be significant, we also allow the possibility of a linear space-charge correction when calculating trajectories. The motivation behind this modeling is the possibility of using the optimization programs available in designing electron optical devices such as electron microscopes.

In our present model we propose to model the effect of the plasma sheath on the particle trajactories by a simple planar boundary at some location behind the plasma electrode. In general it is expected that the position of this plane will vary with current density. Future investigations will look at the possibility of improving the model by using a curved surface.

The validity of this model will be investigated by comparing the model predictions with the twodimensional results from the program SNow 6 .

\section{MODEL GEOMETRY}

Figure 1 shows the geometry used. In these first calculations, the geometry is simple to minimize complications in interpreting results. The beam is assumed to be inserted at a distance $L$ behind the plasma electrode. In the sNow calculation this corresponds to the point at which the initial beam is injected into the plasma. In our model $\mathrm{L}$ corresponds to the position of a planar plate at a potential $\mathrm{V}_{0}$. Typically, L varied between -3.6 and $-0.8 \mathrm{~mm}$. The plasma electrode is $0.2 \mathrm{~mm}$ thick with an aperture radius (a) of $2.5 \mathrm{~mm}$. The extractor is a solid plate located a distance $8 \mathrm{~mm}$ from the upstream face of the plasma electrode. Since we are interested only in the 
behavior of the beam as it leaves the plasma electrode, the emittance was measured at a position $0.4 \mathrm{~mm}$ down stream of the backface of the plasma electrode. Future calculations may move this position further downstream to a region in which the equipotentials are not changing as rapidly.

In the SNOW calculations current densities of 50, $100,200,400$, and $600 \mathrm{~mA} / \mathrm{cm}^{2}$ were used and the extractor voltage was fixed at $41 \mathrm{kV}$. The electron temperature and the incident ion energy was $5 \mathrm{eV}$. The Laplacian solution of this geometry was obtained using the computer code SIMION ${ }^{7}$.

\section{SNOW RESULTS}

Figure 2 shows a typical trajectory and potential distribution obtained from SNOW. A key observation of the trajectory data is that inside the plasma the particles are shielded form each other by the Boltzman electron distribution and thus they move in a straight line until they encounter the plasma sheath. At the sheath, the electron density rapidly decreases, and the particles begin to experience the potential

distribution from the plasma aperture and the extractor electrode. Even though the particles experience this potential, they move a significant axial distance before they begin curve. In addition, those trajectories near the axis of the source are the first to experience a decrease in electron density; particles at larger radius experience this decrease further downstream.

Figure 3 shows the axial position of the $5-\mathrm{eV}$ equi-potential line for the current densities used in the sNow calculations. This potential corresponds roughly to the point at which the electron density begins to decrease rapidly. As expected, a decrease in current density causes the plasma to recede from the plasma aperture. Figure 4 shows the (R', R) emittance diagrams for the various current densities. In Fig. 4 those rays which originate at a radial position less than $2.5 \mathrm{~mm}$ have darkened symbols while those rays with initial radial positions greater than $2.5 \mathrm{~mm}$ are shown by the open symbols. 
In this model we assume that the Poisson potential can be approximated by a Laplacian potential obtained from using the same geometry as in the Poisson solution but replacing the plasma and the plasma sheath by a planar plate located a distance I behind the plasmaelectrode. The potential of the plate is assumed to be potential of the center of the plasma used in SNOW. A first estimate of the value of $L$ is sheath position in SNOW, however, we can also view L as an arbitrary parameter that can be varied to obtain the best fit with the SNOW data. The particle trajectories are calculated from the Laplacian potentials generated by SIMION. The particles are assumed to be emitted perpendicular to the entrance plane with an energy corresponding to the ion energy used in SNOW ( $5 \mathrm{eV}$ ). No correction is made for space charge.

Figure 5 shows the trajectories and the Laplacian voltage distribution for the case in which $L=-2.1 \mathrm{~mm}$. Figure 6 shows the emittance diagrams for various choices of L. Again darken symbols correspond to particles injected with radial positions less than 2.5 $\mathrm{mm}$. In general the shapes of these curves are not that different from those calculated in SNOW. In fact for each current density in Fig. 3 it is possible to obtain an $L$ that gives good agreement with the SNOW results over a relatively large range of the emittance curve.

Figure 7 shows a comparison between the SNOW emittance data and the Laplacian model data for the 200 $\mathrm{mA} / \mathrm{cm}^{2}$ case for two different $\mathrm{L}^{\prime} \mathrm{s}$. The best agreement for small radii occurs at an $L$ of $-2.1 \mathrm{~mm}$ while the best agreement for larger radii corresponds to $L=-1.6$ $\mathrm{mm}$. For the $200 \mathrm{~mA} / \mathrm{cm}^{2}$ case, the sheath position in SNOW was $-1.6 \mathrm{~mm}$. The agreement between this simple Laplacian model and SNOW suggests that the potential distribution produced by the plasma aperture dominates the emittance of the resulting beam and that the effect of the plasma sheath is much smaller.

\section{LAPLACIAN MODEL WITH LINEAR SPACE CHARGE}

The next obvious step is to add a linear spacecharge term to the trajectory calculation. To do this correctly in a region of acceleration, it is necessary to calculate both a radial and an axial space-charge force. As a first step, however, we present a series of calculations in which the axial space-charge force 
is ignored and only a radial space-charge force term is used in the trajectory equations. The form of the radial electric field due to space charge is

$$
E_{r}^{S C}(z)=\frac{r J o}{2 \varepsilon c \sqrt{T o+\Phi(0, z)}},
$$

where $r$ is the radius of the trajectory, Jo is the beam current density, To is the initial beam energy, $\varepsilon$ is the electrical permitivity, $c$ the velocity of light and $\Phi$ is the potential along the $z$-axis for $r=0$.

Figure 8 shows the trajectories for this calculation and the potentials corresponding to an $L$ of $-2.6 \mathrm{~mm}$. Figure 9 shows the corresponding emittance diagrams. The heavy solid line is the sNow calculation for $200 \mathrm{~mA} / \mathrm{cm}^{2}$; the three lighter lines correspond to three different $I$ positions. The symbols show the no space-charge calculation for an $L$ of $-2.6 \mathrm{~mm}$. Since only the radial part of the space-charge force has been used, it is not clear if the good agreement is fortuitous or not. The results do show that adding a linear space-charge term can significantly improve the accuracy of the models. If the good agreement between this model and SNOW is maintained with the additional of the axial space-charge term, then these results indicate that the contribution of the non-linear part of the space-charge to emittance growth is small.

\section{CONCLUSION}

We have compared the emittance calculations of SNOW with a simple model that replaces the Poisson potential with a Laplacian potential calculated using the original source geometry but with the plasma replaced by a simple planar plate. In our model we have also looked at the effect of including a linear space charge correction in the radial direction. With this model there is only one free parameter L. In general, the emittance curves from this simple model are in good agreement with those calculated from SNOW.

This good agreement suggests that it would be worthwhile to pursue this model further. In particular, the axial space-charge correction needs to 
be implemented and tested and the results need to be compared with more complex geometries. If the results of this comparison are still positive, then the computer codes and the associated techniques that are used to minimize electron aberrations in electron microscopes may be useful in optimizing the optics of ion sources.

\section{REFERENCES}

1. P. Spadtke, "Computer Modeling", in The Physics and Technology of Ion Sources, I.G. Brown, editor, (Wiley\&Sons, 1989) p. 107.

2. J. H. Whealton, "Computer Modeling of Negative Ion Beam Formation," J. Appl. Phys. 64(11), 6210 (1988).

3. E. Thompson, "The Design of Low Divergence Ion Source Extraction Systems," Particle Accelerators 4,69 (1972).

A.J.T. Holmes, and E. Thompson, "Beam Steering in Tetrode Extraction Systems," Rev. Sci. Instrum. $52(2), 172$ (1981).

4. A.J.T. Holmes, and E. Surrey, "Beam Emittance Growth Caused by Non-Uniform Ion Density," Rev. Sci. Instrum. 62(5), 1173 (1991).

5. E. Surrey and A.J.T. Holmes, "The Effects of Ion Beam Optics of a Spherical Aberration at the Plasma Boundary," Rev. Sci. Instrum. 61(8), 2171 (1990).

6. J. E. Boers, SNOW, Thunderbird Simulations, 626 Bradfield Drive, Garland Texas 75042-6005 (214272-5858)

7. D. A. Dahl, and J.E. Delmore, "The SIMION PC/PS2 User's Manual Version 4.0," EGG-CS-7233, 1988

\section{DISCLAIMER}

This report was prepared as an account of work sponsored by an agency of the United States Government. Neither the United States Government nor any agency thereof, nor any of their employees, makes any warranty, express or implied, or assumes any legal liability or responsibility for the accuracy, completeness, or usefulness of any information, apparatus, product, or process disclosed, or represents that its use would not infringe privately owned rights. Reference herein to any specific commercial product, process, or service by trade name, trademark, manufacturer, or otherwise does not necessarily constitute or imply its endorsement, recommendation, or favoring by the United States Government or any agency thereof. The views and opinions of authors expressed herein do not necessarily staic or refiect those of the United States Government or any agency thereof. 


\section{MODEL GEOMETRY}

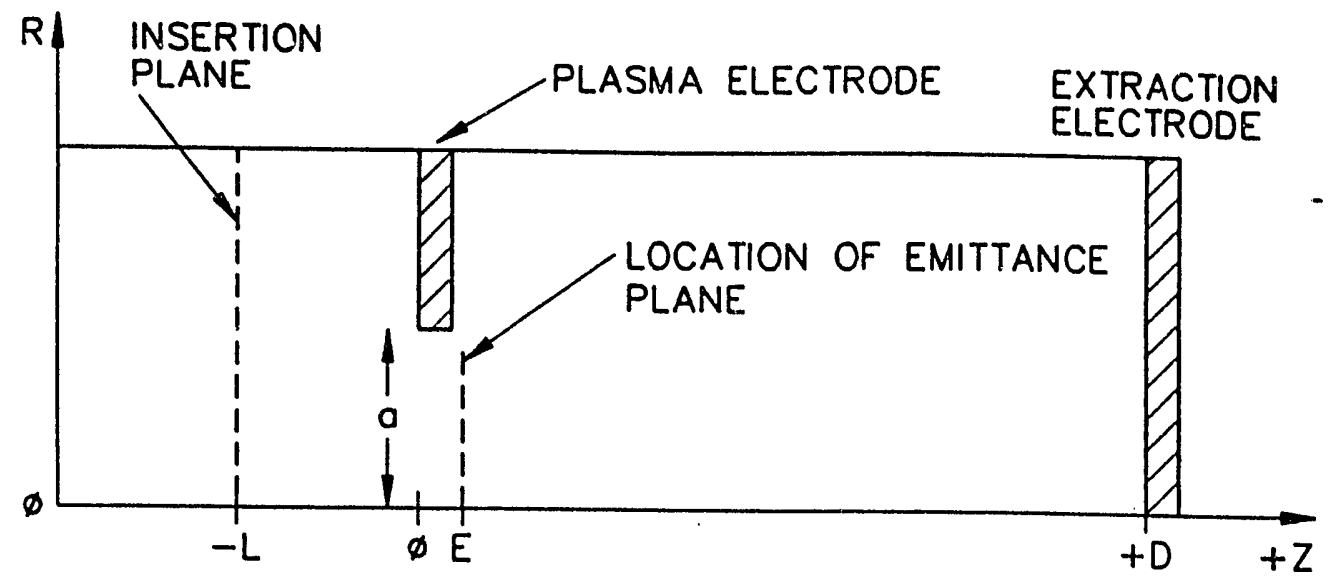

Figure 1. Geometry Used in Source Calculations.

$\nabla=5$ OV POSITION VERSUS CURRENT DENSITY

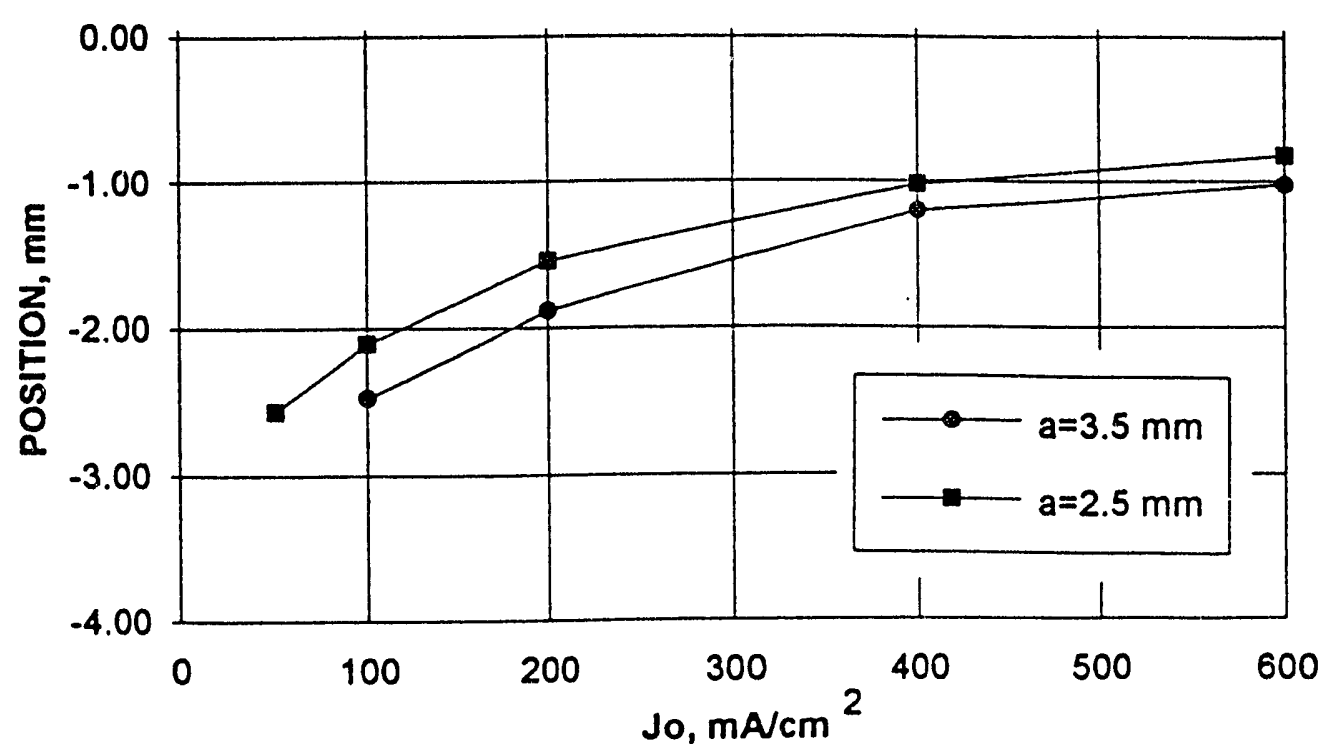

Figure 2. Axial Position of Sheath in the SNOW Calculations Versus Current Density 

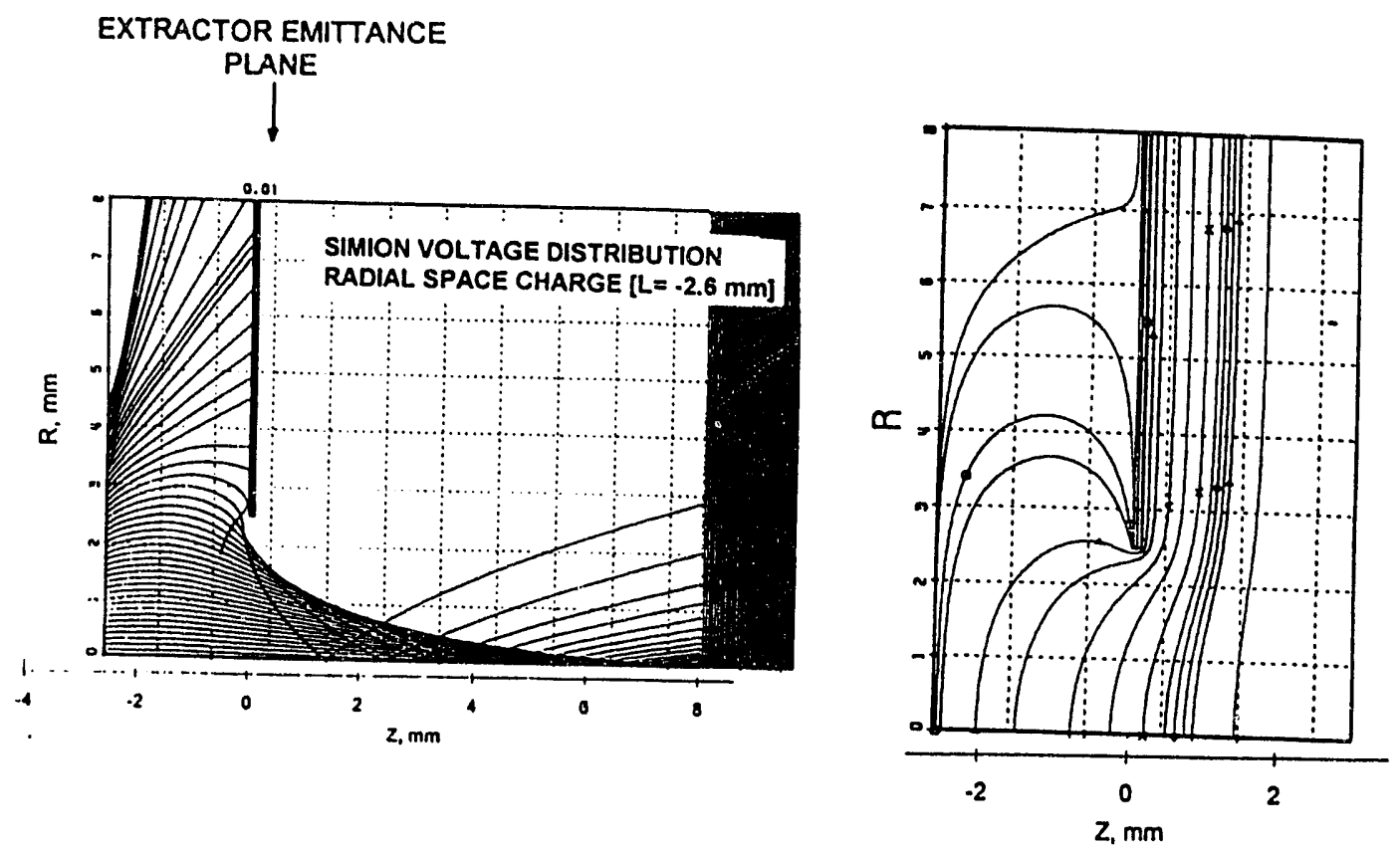

Figure 3. Trajectories and Potentials for the SNOW Calculation.

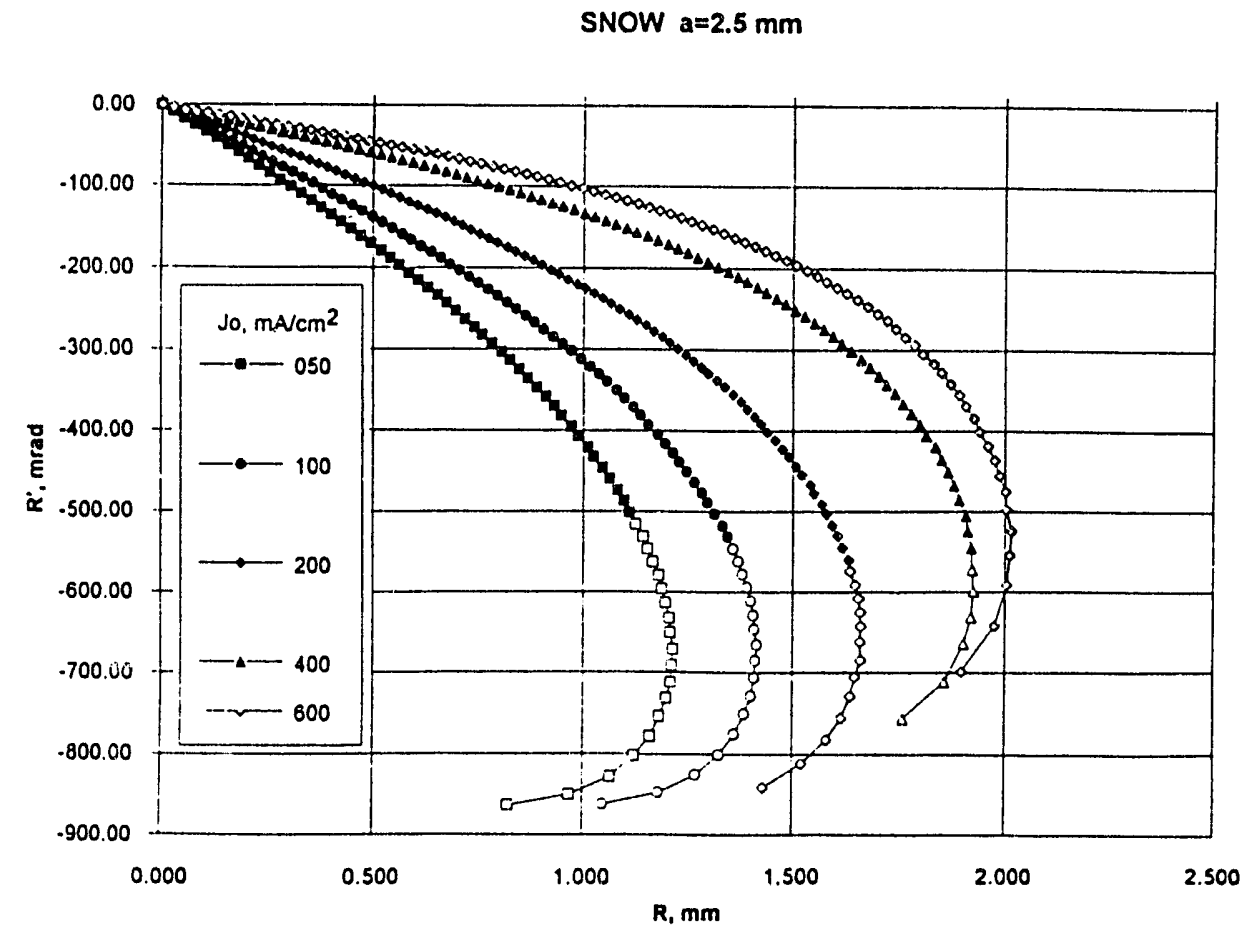

Figure 4. Emittance Curves for Various Current Densities from SNOW. 

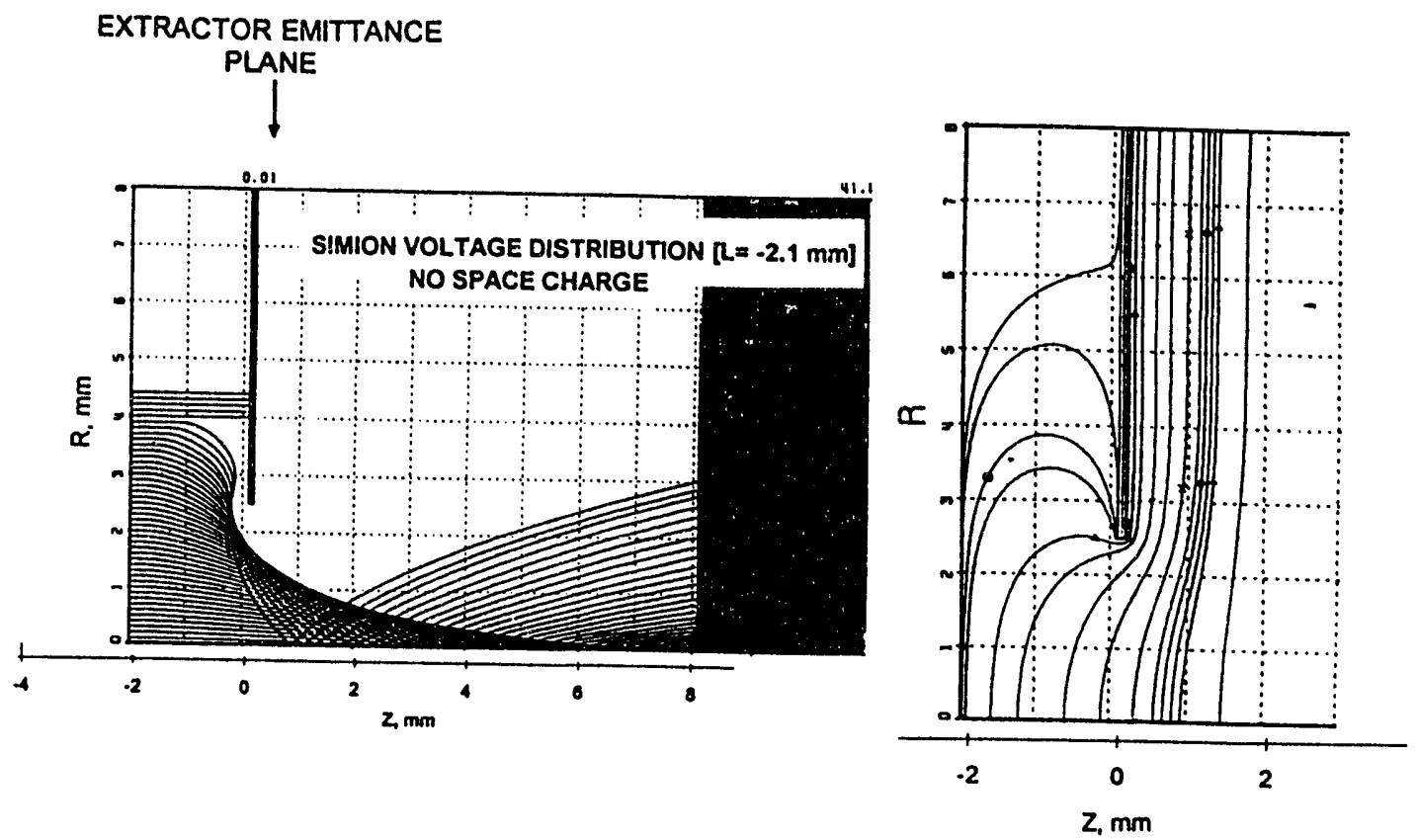

Figure 5. Trajectories and Potentials for the SIMION Potential Distribution for an $\mathrm{L}$ of $-2.1 \mathrm{~mm}$ and No Space Charge.

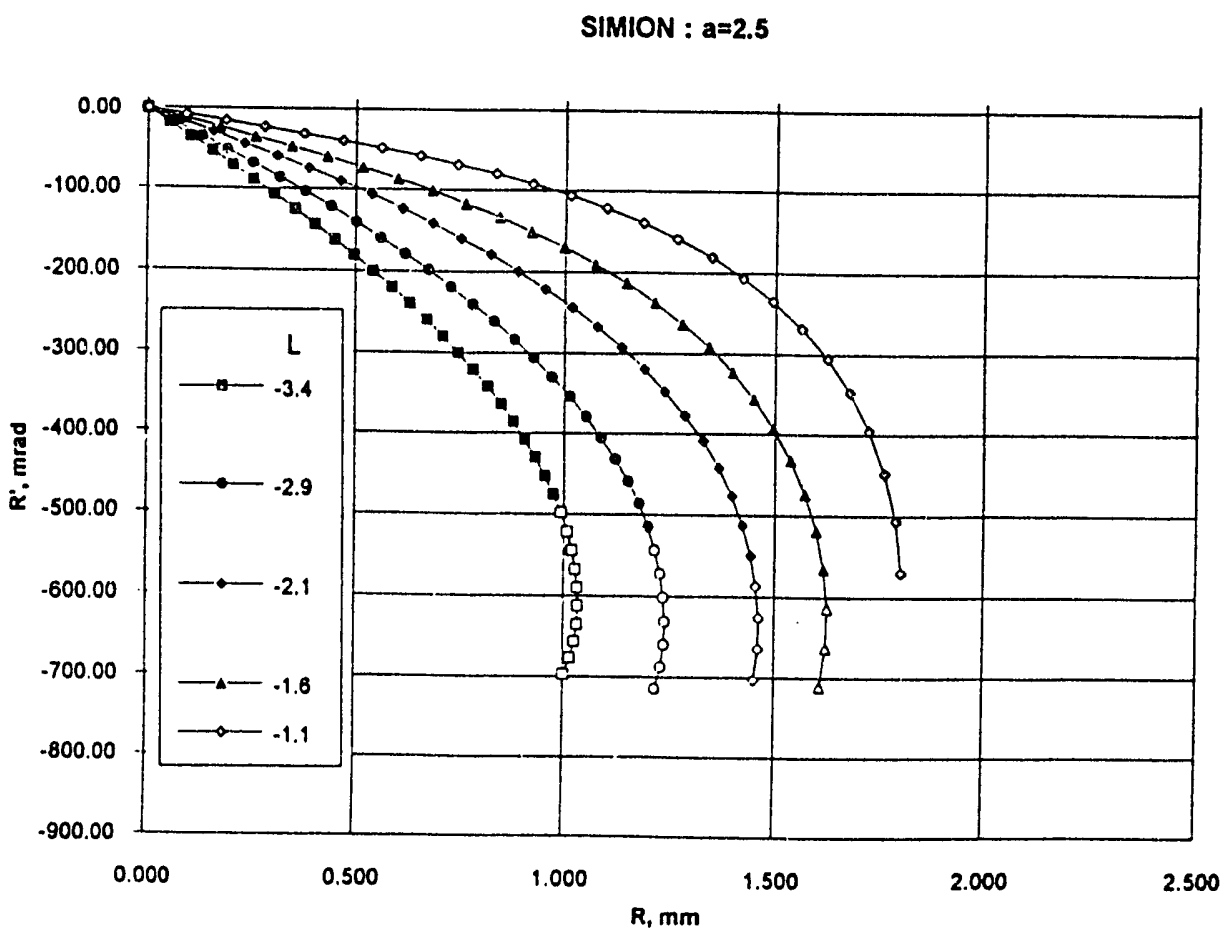

Figure 6. Model Emittance Curves for Various L Positions. 


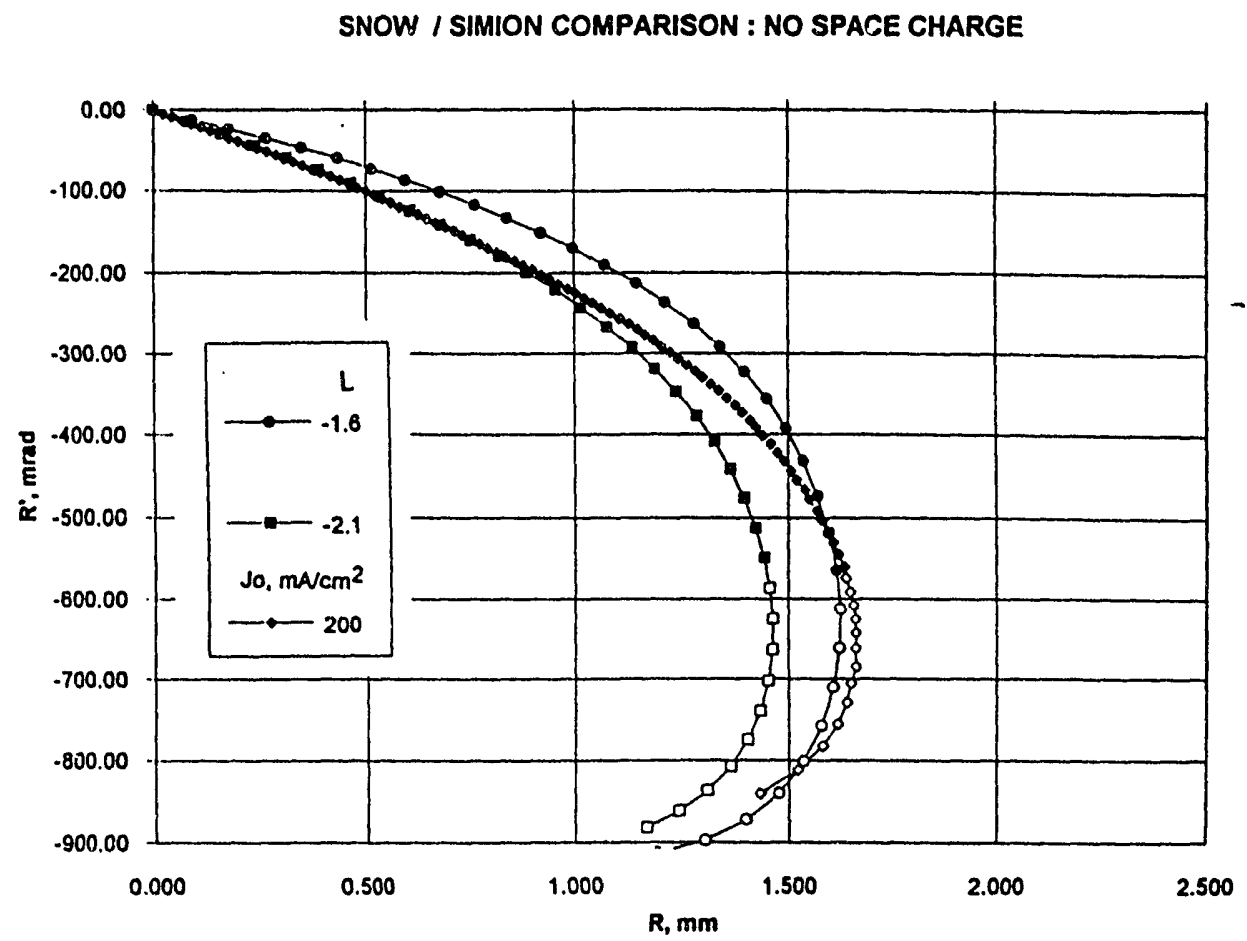

Figure 7. Comparison of the SNOW Emittance Curve and the Model Emittance Curves for Two Different $L$.
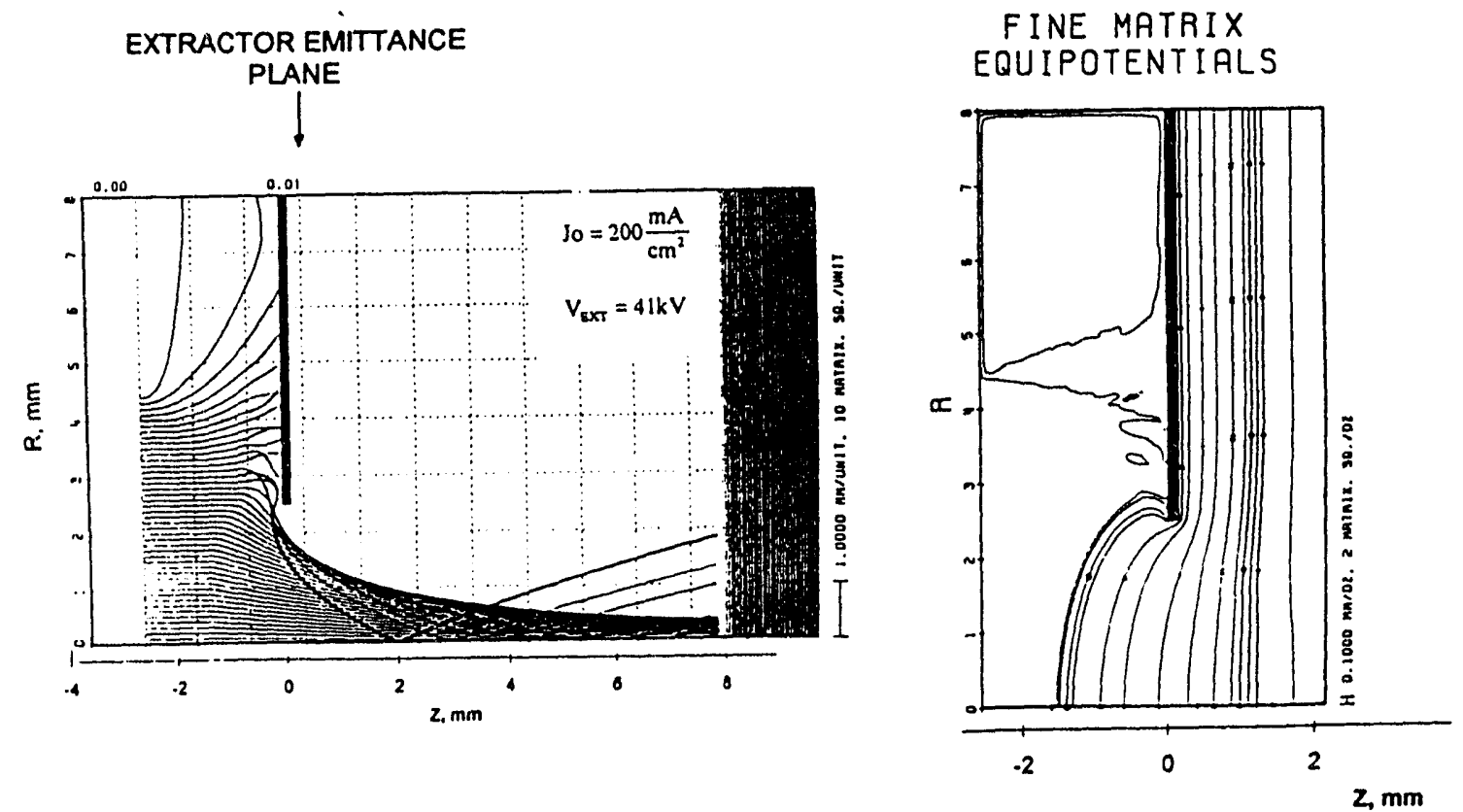

Figure 8. Trajectories and Potentials for the SIMION Potential Distribution for an $\mathrm{L}$ of $-2.6 \mathrm{~mm}$ and with a Radial Space-Charge Correction. 


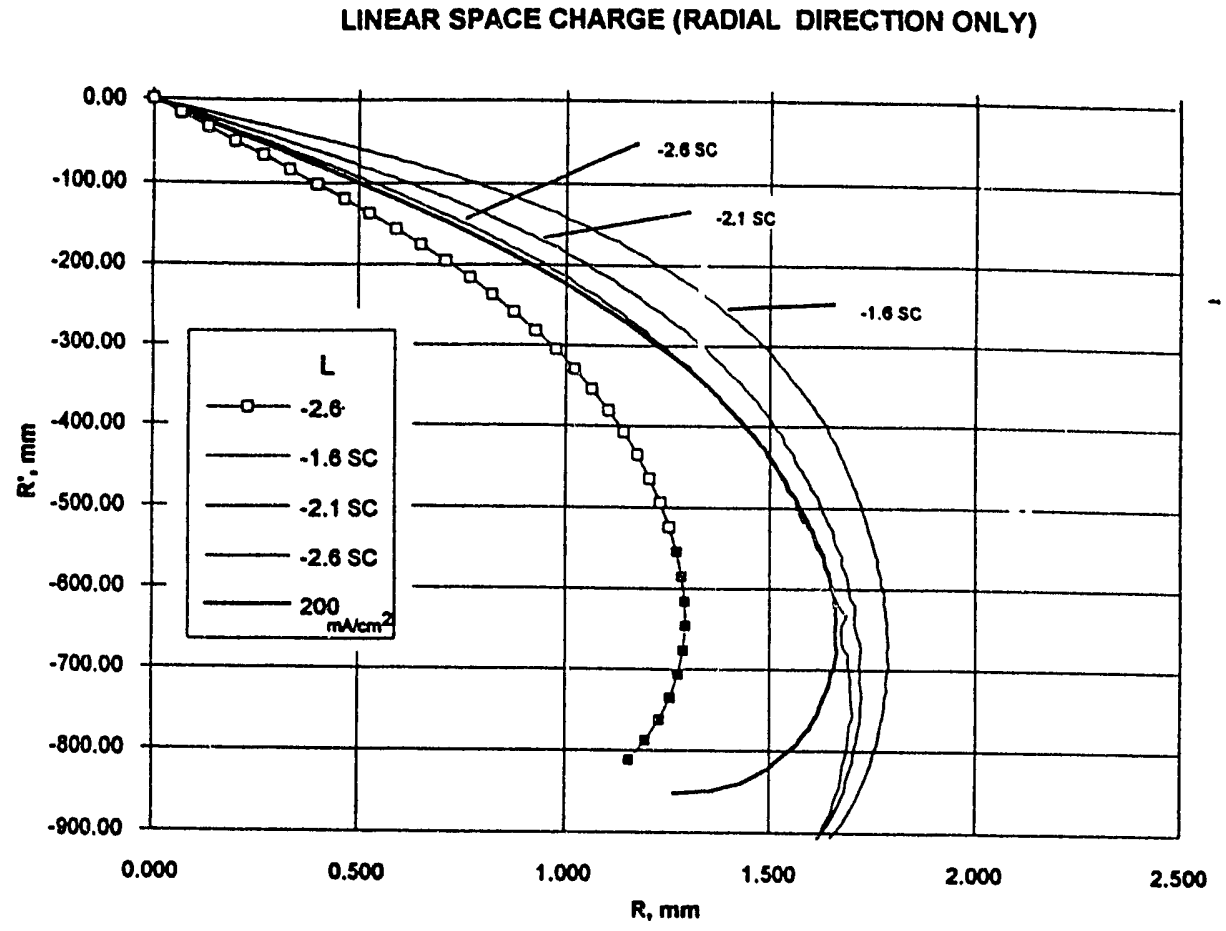

Figure 9. Comparison of the SNOW Emittance Curve and Three Different Model Calculations with Radial Space Charge. The Symbols correspond to a Model Calculation with No Space Charge. 

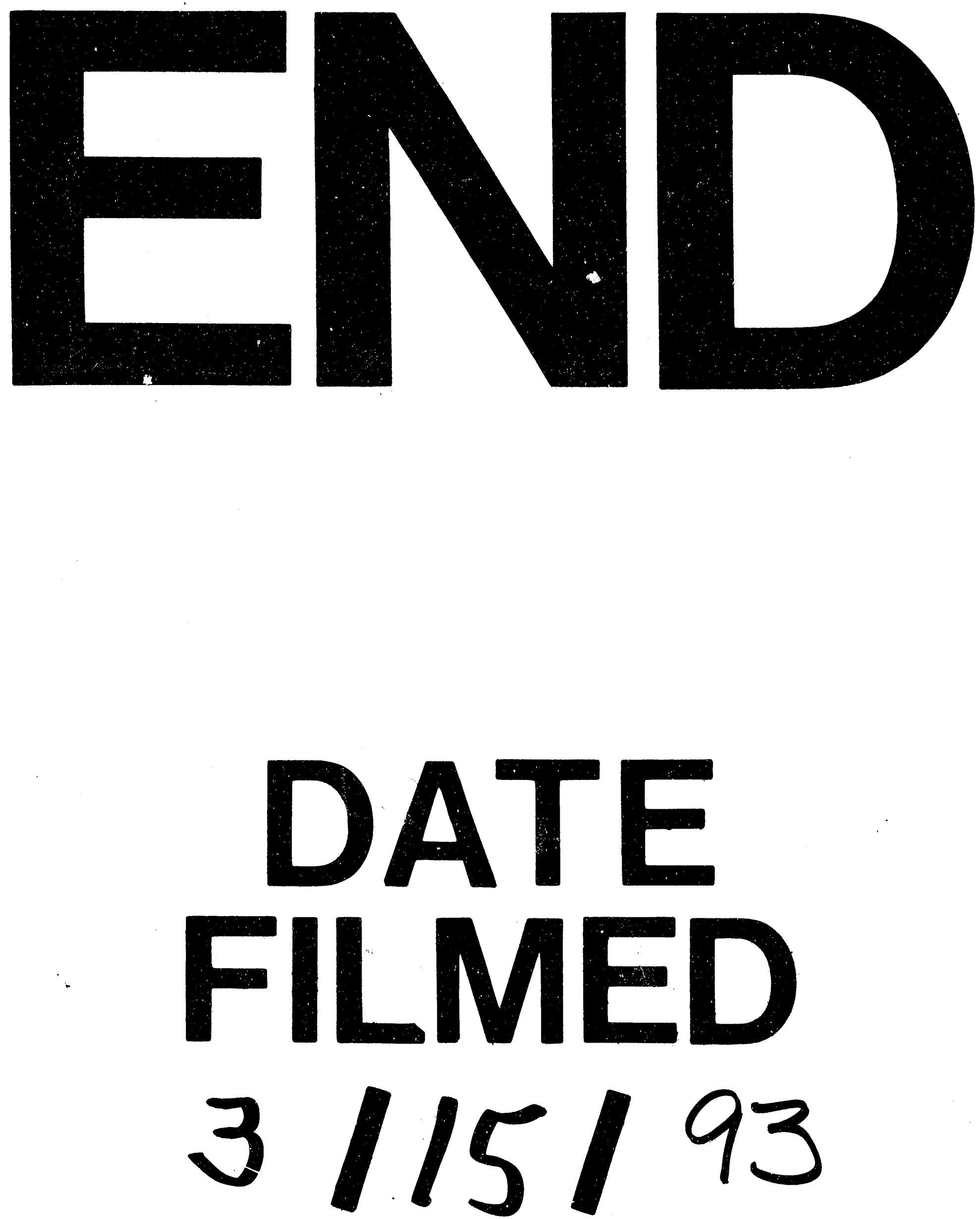
